\title{
La fabrique symbolique de la femme et de l'homme en ses espaces
}

Représentations poétiques et pratiques rituelles (Athéna et Aphrodite)

The symbolic fabrication of woman and man in its spaces : poetic

representations and ritual practices (Athena and Aphrodite)

\section{Claude Calame}

\section{(2) OpenEdition Journals}

Édition électronique

URL : http://journals.openedition.org/ress/3252

DOI : $10.4000 /$ ress.3252

ISBN : $1663-4446$

ISSN : 1663-4446

Éditeur

Librairie Droz

Édition imprimée

Date de publication : 15 novembre 2015

Pagination : $51-74$

ISSN : 0048-8046

Référence électronique

Claude Calame, «La fabrique symbolique de la femme et de l'homme en ses espaces », Revue européenne des sciences sociales [En ligne], 53-2 | 2015, mis en ligne le 15 novembre 2018, consulté le 03 mai 2019. URL : http://journals.openedition.org/ress/3252 ; DOI : 10.4000/ress.3252 


\title{
LA FABRIQUE SYMBOLIQUE DE LA FEMME ET DE L'HOMME EN SES ESPACES REPRÉSENTATIONS POÉTIQUES ET PRATIQUES RITUELLES (ATHÉNA ET APHRODITE)*
}

\author{
CLAUDE CALAME
}

EHESS, Paris

claude.calame@unil.ch

\begin{abstract}
Résumé. S'il est un espace construit qui peut apparaitre comme réalité géo-architecturale, mais qui offre une configuration d'ordre symbolique, c'est bien le sanctuaire, en particulier en Grèce ancienne. Imaginé, configuré et dit par l'homme de culture en rapport avec les divinités qu'il vénère, l'espace du sanctuaire retracé par les archéologues comme ensemble de realia requiert une perspective d'anthropologie culturelle (historique). Avec une telle approche, il est possible d'identifier les valeurs symboliques attribuées à un espace paysager, d'une part en relation avec les actions narratives que le «mythe» y met en scène dans le passé héroïque, et d'autre part en rapport avec les pratiques sociales et rituelles que cet espace architectural accueille, hic et nunc, dans un double processus d'anthropopoiésis, narrative et rituelle. C'est ce que l'on aimerait montrer à l'exemple d'un complexe de sanctuaires paysagers qui, sur l'Acropole d'Athènes, disent, entre mythes et rituels, les valeurs symboliques attachées au statut de l'épouse de citoyen.
\end{abstract}

Mots-clés: anthropopoiésis, arrhéphores, Athènes, espaces cultuels, espaces paysagers, identités de sexe.

\begin{abstract}
If there exists a constructed space that beyond its geo-architectural reality refers to a symbolic order, it is the case of the sanctuary, in particular the one of Ancient Greece. Imagined, configured and designated by the man of culture in relation to the divinities he venerates, the sanctuary as a place defined by the archeologists as a set of realia needs to be considered from a (historical) cultural anthropology perspective. In such a way, it is possible to identify the symbolic values attached to any landscape area in relation to the narrative actions staged in the heroic past, on the one hand, and with social and ritual practices that take place in this architectural space, hic et nunc, on the other, along a double process of ritual and narrative anthropopoiesis. This article aims to illustrate such a process with the example of a complex of landscape sancturaries on the Athenian Acropolis, that expresses, between myths and rituals, the symbolic values attached to the citizen's wife status.
\end{abstract}

Keywords: anthropopoiesis, arrhephores, Athens, cultural spaces, landescape spaces, sexual identity.

"Cet article est une version révisée de Claude CALAME, 2010, «Jardins cultuels et rites féminins d'adolescence. L'autochtonie athénienne en ses sanctuaires paysagers», Revue de l'histoire des religions, 227-4, p. 459-479. 
Si en histoire d'une culture autre il est une discipline qui sattache aux realia et à leur reconstitution, c'est certainement l'archéologie. L'investigation archéologique porte en général sur des espaces construits. Or ces espaces correspondent à des realia qui d'une part procèdent d'intentions et de configurations culturelles, qui d’autre part étaient les lieux de pratiques et les objets de représentations hautement symboliques. C’est en particulier le cas des espaces de déploiement de la culture polythéiste de la Grèce ancienne, domaine d'une archéologie qui s'est illustrée dans la reconstitution de grands sanctuaires : Olympie, Delphes, l’Acropole d'Athènes. Mais avec quelles significations, pour quelles pratiques?

De plus, d'«Homère » à Callimaque en passant par Pindare ou Sophocle, la culture de la Grèce ancienne, en tant que «song culture», se distingue par ses productions poétiques. Elles abondent en descriptions d'espaces chargés de valeurs symboliques dans une construction partagée entre réalité géographique et architecturale, et figuration poétique.

Commençons par une description célèbre de l’Odyssée :

Autour de la grotte croissait un bois luxuriant, aunes, peupliers, cyprès odorants.

Là nichaient des oiseaux aux ailes bandées, chouettes, éperviers, corneilles de mer, bavardes, qui se plaisent aux vols marins. À l'entrée de la grotte creusée dans le roc se déployait un plant de vigne en pleine vigueur, chargé de grappes. Voisines les unes des autres, s'écoulant de-ci de-là, quatre sources faisaient surgir une eau transparente; tout autour de tendres prairies où fleurissaient la violette et l'ache. Parvenant en un tel lieu, un immortel lui-même resterait stupéfait, regard et cœur sous le charme'. 
Même s'il est centré sur l'espace «naturel» qu'est pour nous une grotte, l'environnement de ce site poétique est entièrement composé: pas de «nature » à l'état primitif ou à l'état pur, mais une configuration d'éléments végétaux qui, culturellement et poétiquement, évoquent un érotisme féminin teinté d'une aspiration au monde de l'au-delà. Si sur l'aune nous n'avons pas de renseignements quant à la valeur culturelle que lui attribuaient les Grecs, le peuplier noir se dresse, par exemple, auprès de la prairie touffue sur laquelle Phèdre, desséchée par la passion délirante voulue par Aphrodite, aimerait trouver le repos en étanchant sa soif amoureuse à l'eau pure d'une source; quant au cyprès il peut servir de repère lumineux sur la voie qui conduit les défunts à travers l'obscurité humide de l'Hadès vers le domaine protégé par Perséphone - prairie et bois sacré ${ }^{2}$. Évoquant Dionysos et Artémis respectivement, vrilles de la vigne et ruisseaux d'eau claire nous ramènent à la prairie, une praire émaillée de violettes et de fleurs d'ache; la violette s'inscrit dans le paradigme des fleurs aux parfums séducteurs qui couvrent la «tendre prairie » d'où la jeune Perséphone est enlevée pour être entraînée dans l'Hadès comme épouse de Pluton; quant au céleri il est associé à la violette et à la rose, la fleur d'Aphrodite, dans une brève chanson populaire d'inspiration certainement érotique ${ }^{3}$. Si un immortel lui-même se laisserait saisir et charmer par un tel lieu, c'est qu'il évoque l'immortalité ; et ceci par l'intermédiaire des deux états qui peuvent mettre le mortel hors de lui-même : l'amour érotique et le sommeil de la mort elle-même. D’un côté Éros qui vous ravit par le pouvoir du désir, de l'autre Thanatos qui peut vous transporter, dûment initié, vers les îles de bienheureux. La prairie évoque le bonheur divin et l'immortalité dans une sorte de retour à un espace de l'âge d'or ; mais c'est une immortalité dont la promesse reste pour les humains profondément ambivalente. Construit par l'homme, revisité par la pratique poétique, l'espace paysager porte le reflet de son identité, de l'identité que symboliquement il se donne.

2 Euripide, Hippolyte 208-21I; lamelle d'or d'Hipponion, Orphica fr. 474, 3 Bernabé avec les nombreuses références bibliographiques données dans Calame, 2006a, p. 234-246.

3 Hymne homérique à Déméter I-II ; carm. pop. fr. 852 Page; voir Calame, 2002, p. I74-I85 pour d'autres références. 
L'espace construit, imaginé et dit par l'homme en rapport avec les divinités qu'il vénère requiert donc une perspective d'anthropologie culturelle (historique). Avec une telle approche il est possible d'identifier les valeurs symboliques attribuées à un espace paysager d'une part en relation avec les actions narratives que le «mythe» y met en scène dans le passé héroïque, et d’autre part en rapport avec les pratiques sociales et rituelles qui s'y déroulent, hic et nunc. Espaces construits qui nous apparaissent comme des realia, sans doute, mais que pratiques rituelles et récits poétiques configurent sur le mode symbolique de l'expression de valeurs culturelles.

C’est dire qu'en particulier les espaces aménagés en jardins doivent être considérés dans leurs valeurs propres et dans leurs fonctions indigènes, en relation avec celles et ceux qui les perçoivent, les modèlent, les habitent et, surtout, les pratiquent. Ces espaces exigent donc de nous, à partir d'une réalité de géographie physique, un effort rigoureux de traduction transculturelle, parfois en comparaison anthropologique avec d'autres cultures. À l'exemple d'espaces consacrés à une divinité et des manières de les dire tout en les pratiquant, il s'agit de faire apparaître les spécificités de la conception partagée de l'homme et les singularités des pratiques sociales et religieuses qui fondent l’aménagement du territoire dans une culture aussi distante que l'est de nous la culture grecque antique ${ }^{4}$.

\section{I.PRAIRIES DE POĖTES ET JARDINS DE CULTE}

En effet, avec les connotations que lui attribue la création poétique homérique, la caverne arborée s’avère habitée par une femme séductrice: donc pas de «wilderness » dans le monde de l'Odyssée! La grotte d'amour et de mort immortalisante est la demeure de la nymphe Calypso. La divinité qui partage avec Hermès nectar et ambroisie et Ulysse la décrit comme «une déesse qui inspire l'émerveillement, mais à la voix humaine $»^{5}$. Par les moyens enchanteurs de l'amour, la nymphe aux belles boucles et aux légers vêtements entend faire d’Ulysse, désormais privé de ses

4 Pour la question complexe de la traduction anthropologique en termes «etic» des valeurs symboliques «emic» d'une autre culture, je renvoie à ma contribution (2002).

5 Homère, Odyssée 5, 92-94 et 12, 449, voir aussi 9, 29. 
compagnons, un immortel en le soustrayant à la vieillesse; mais par la volonté de Zeus, Ulysse doit accomplir son destin de mortel et rejoindre les siens à Ithaque. En relation avec les qualités enchanteresses de celle qui l'habite, le paysage boisé et fleuri qui accueille Calypso s'avère être un espace de transition : transition potentielle vers l'immortalité et donc vers l'état divin; transition narrative pour Ulysse, privé de tout lien social avec ses pairs mortels, vers la communauté semi-divine et utopique des Phéaciens; puis retour à la cour d'Ithaque, avec sa sociabilité de mortels selon la représentation (grecque) de la civilisation des hommes.

Donc pas de sentiment grec pour le paysage en tant que tel ${ }^{6}$, mais des configurations narratives et poétiques qui établissent des correspondances entre les valeurs métaphoriques évoquées par les éléments qui le composent et les qualités de ses occupants qui sont aussi des sujets d'action: dans un paysage entièrement composé d'éléments suscitant pour la représentation grecque le désir amoureux, la divine Calypso tente d'immortaliser, par le biais d'Éros, Ulysse le mortel. Ce qui nous apparaît comme un paysage est donc le lieu d'une action poético-narrative qui correspond à un passage (manqué). Dans ce changement de statut, l'amour interfère avec la mort comme c'est souvent le cas dans les représentations hellènes de l'état second provoqué par Éros. L’adolescent ailé est en effet l'incarnation du désir érotique qui vous envahit pour vous mettre hors de vous-même. Par référence à deux études précédentes, rappelons ici simplement les affinités qui associent, du point de vue paysager, les prairies fleuries évoquant les pouvoirs séducteurs d’Aphrodite et les prés élyséens quau-delà de l’Hadès protège Perséphone. D’une part le sanctuaire-jardin dans lequel des vers composés par Sappho invitent Aphrodite à intervenir pour partager avec ses jeunes servantes nectar et réjouissances musicales; de l'autre les prairies et les bois sacrés où Perséphone accueille les âmes qui sont porteuses des textes rituels et poétiques transcrits sur des lamelles funéraires d’or, véritables passeports pour l'au-delà7.

6 Sur les représentations grecques de ce qui est pour nous le paysage, voir la contribution de Cohen, 2007; pour les usages du paysage dans mythe et cultes grecs, voir le bon chapitre de Buxton, 1994, p.80-113.

7 Sappho fr. 2 Voigt et Lamelle de Thurii A 4 Zuntz = Orphica fr. 487 Bernabé ; voir Calame, 2006a, p. 263-27l et 2006b, avec les références bibliographiques sur deux textes désormais surcommentés. 
Si la prairie de Perséphone relève d'une représentation poétique que l'on retrouve par exemple chez Pindare, le sanctuaire paysager décrit par Sappho correspond certainement à un lieu de culte réel. Dans les deux cas, c'est la récitation ou le chant des vers concernés qui permet d’accomplir le passage indiqué : poèmes de culte dont la performance contribue à l'accomplissement rituel du passage spatial et au changement de statut- initiation cultuelle aux plaisirs d'Aphrodite pour la poésie de Sappho, accès rituel à une forme d'immortalité pour les bienheureux initiés méritant la compagnie de Perséphone pour l'éternité. Quant à l'environnement paysager de la grotte de Calypso, il relève d'une composition poétique qui intègre des représentations culturelles et qui est subordonnée à la narration du retour d’Ulysse vers le monde des mortels incarné par Ithaque.

Appelant la présence d'Aphrodite dans le sanctuaire-jardin qui lui est consacré, le poème de Sappho nous invite donc à partager avec la déesse des espaces paysagers bien réels, des espaces construits, des espaces de culte, des espaces symboliquement fortement chargés où s'accomplit un passage rituel. Ce sont des espaces d'architecture essentiellement végétale qui accueillent une action cultuelle liée à la transition d'un état à un autre, à ce qu'on appelle en anthropologie un rite de passage. Dans le régime polythéiste propre à chaque cité grecque, le changement rituel de statut et par conséquent la transformation identitaire impliquent l'intervention d’une divinité spécifique, en général en collaboration avec un héros tutélaire et d'autres divinités parentes ${ }^{8}$. Par l'intermédiaire des récits qui expliquent l'origine de la pratique rituelle et par métaphores interposées, nombreuses sont les qualités partagées entre la divinité agente du passage, les acteurs du récit étiologique qui fonde l’acte rituel, le statut des protagonistes de la transition et le contexte d’architecture végétale qui accueille cet acte cultuel, avec les valeurs culturelles et symboliques attachées aux végétaux concernés.

8 Sur les différentes formes assumées par les rites de passage dans les cités grecques en particulier en ce qui concerne les pratiques de l'initiation tribale réservée aux adolescents ou aux adolescentes, voir désormais les études critiques réunies dans Padilla (dir.), 1999 (notamment la mienne, reprise et développée en français dans Affergan et al., 2003, p. 127-173) ainsi que dans Dodd et Faraone (dir.), 2003. Le concept d'«anthropopoiésis» comme construction pratique et culturelle de l'humain a été développée dans différentes études collectives dont Remotti (2003) fait état; pour une interprétation anthropopoiétique du cursus initiatique, rituel et poétique, destiné aux jeunes filles de Sparte, voir mon étude (2013). 
De ce point de vue de la configuration architecturale et symbolique d'espaces légitimés par des récits et accueillant des actes rituels relatifs au changement collectif de statut, l'espace cultuel athénien est particulièrement riche de sanctuaires paysagers; ils sont les lieux de différents rites de passage destinés en particulier aux jeunes filles. L’organisation physique de ces sanctuaires nous confronte à une «landscape architecture » concrète; cette architecture paysagère sert de cadre pratique et symbolique à différents processus relevant de ce que l'on qualifiera comme «anthropopoéisis » : fabrication culturelle et sociale de l'être humain, homme et femme, avec une identité statutaire et symbolique spécifique. En l'occurrence les pratiques anthropopoiétiques que constituent les rites de passage accomplis dans des espaces d'architecture paysagère à haute teneur symbolique sont adossées aussi bien à une mémoire narrative et poétique qu’à des actes collectifs animés par les mêmes représentations symboliques. D’ordre aussi bien discursif que rituel, ces processus anthropopoiétiques sont traversés en particulier par les valeurs métaphoriques attribuées aux éléments du paysage architectural et cultuel qui les accueille; par l'autorité d'une divinité, par l'exemple héroïque et par la pratique rituelle, ils définissent et ils réalisent des identités politiques (au sens grec et large du terme) répondant en particulier au critère du gender compris comme ensemble des identités et des relations pratiques et symboliques marquées par le sexe.

\section{ENTRE RITUEL ET MYTHE Athéna et Aphrodite}

À commencer par Aphrodite, et par Aphrodite aux Jardins. Le sanctuaire athénien a été identifié grâce à une dédicace à Éros sur une terrasse située dans le flanc de la falaise septentrionale de l'Acropole; les traces archéologiques en sont à vrai dire peu indicielles. Mais nous avons des textes ${ }^{9}$ !

9 On trouvera chez Pirenne-Delforge (1994, p. 48-62) les quelques rares informations que nous avons sur le sanctuaire et le culte dédiés à Aphrodite aux Jardins. De manière générale, sur les cultes jardiniers rendus à Aphrodite voir Motte, 1973, p. 121-137. 
Ainsi, à l'occasion de sa visite du roc sacré, Pausanias, à la fois historien et géographe de la Grèce des origines, mentionne évidemment la présence de l'olivier d’Athéna. Dans l'enclos aménagé devant le temple d’Athéna Polias (qui est devenu pour nous l'Érechthéion), c'est l'olivier que la déesse avait fait pousser sur l'Acropole dans sa rivalité avec Poséidon pour la possession de l'Attique. Puis, dans l'immédiat voisinage de l'arbre toujours vivace et à l'intérieur de cette enceinte sacrée, le Périégète signale le petit sanctuaire consacré à Pandrosos ; cette héroïne athénienne est la seule des trois filles de Cécrops à ne pas s'être rendue coupable de la faute que nous verrons.

Contrairement à son habitude, le Périégète rapporte à ce propos non pas un récit héroïque, mais les gestes d'une action rituelle. Le rite est accompli par deux des quatre arrhéphores, les très jeunes filles auxquelles était confié le tissage du péplos destiné à Athéna Polias à l'occasion des Panathénées. Peu avant la célébration de cette grande fête civique, les deux filles quittaient de nuit leur demeure située sur l'Acropole en portant sur la tête les objets secrets que leur avait confiés la prêtresse d’Athéna; en empruntant un passage souterrain, elles déposaient ces objets dans le sanctuaire d’Aphrodite «dans les Jardins», adossé au flanc septentrional du rocher de l'Acropole; elles en revenaient en apportant un autre objet, lui aussi soigneusement dérobé à leur regard. À la suite de ce double parcours rituel, les deux arrhéphores concernées laissaient définitivement la maison édifiée à leur intention pour être remplacées par deux autres jeunes filles ${ }^{10}$.

Or cet étrange rituel peut être mis en relation étiologique avec l'un des récits héroïques qui expliquent la délimitation du territoire de l'Attique et la fondation d'Athènes tout en légitimant les prétentions identitaires des Athéniens à l'autochtonie. La légende athénienne raconte en effet la naissance du petit Érichthonios qui va devenir l'un des rois légendaires de la cité. L’enfant surgit des entrailles du sol de l’Attique après que le sperme d’Héphaïstos, dans sa vaine poursuite amoureuse de la vierge Athéna, ait fécondé Gé. Après avoir fait de Terre la mère biologique d'Érichthonios et d'Athéna sa mère symbolique, le

10 Pausanias I, 27, 2-3; pour la bibliographie au sujet de ce rituel, voir infra note 15. 
récit se poursuit avec la courotrophie manquée de l'enfant par deux des trois filles du premier roi autochtone de l'Attique, Cécrops : anthropopoiésis individuelle puisque la pratique relève du récit héroïque.

Mais cet épisode «mythique», sur lequel on va revenir dans le détail, sert sans doute d'explication fondatrice, d'ordre étiologique, pour l'étrange pratique rituelle rapportée par Pausanias. Remarquons pour l'instant que, par l'intervention de la fécondité d'une terre qui apparaît également comme la mère des futurs citoyens autochtones, ce récit s'inscrit dans la saga de la rivalité d’Athéna et de Poséidon pour la délimitation, la définition et le contrôle du territoire de l'Attique. La légende contribue à l'institution et à la définition du pouvoir partagé par les deux futurs dieux tutélaires de la cité d’Athènes tel qu'il était figuré sur le fronton occidental du Parthénon de l'époque classique: d'un coup de son trident, Poséidon faisait surgir du roc de l’Acropole la source d'eau salée dénommée plus tard Mer Éréchthéide et insérée, comme trace de la mémoire civique, dans le futur Érechthéion; Athéna quant à elle y faisait croître le premier olivier qui sera rituellement inclus dans le périmètre cultuel où, auprès du même Érechthéion, s'élèvera le petit sanctuaire héroïque réservé à la Cécropide Pandrosos et mentionné par Pausanias ${ }^{11}$. Ce qui est en jeu ici, ce sont bien les capacités productrices du sol de l'Attique, roc de l'Acropole inclus! Ces virtualités sont illustrées par les gestes symboliques et merveilleux accomplis dans la Cité haute d'Athènes même. Elles sont matériellement inscrites dans un paysage cultuel encore à visiter : olivier d'Athéna et mer Érechthéide surgissant tous deux du compact et aride rocher de la sainte Acropole. L'espace du sanctuaire est modelé selon le récit fondateur, d'ordre anthropopoiétique.

II Le récit est évoqué par Hérodote 8, 55 et par Thucydide 2, 15, I, voir aussi Pseudo-Apollodore, Bibliothèque 3, 14, I (sur Erekhtheìs thálassa); pour la scène représentée sur le fronton occidental du Parthénon et sur les différentes versions d'une rivalité fondatrice, voir Parker, 1987, p. 198-199, et Hurwit, 1999, p. 174-178 et p.292-293. 


\section{VALEURS CULTURELLES ET MÉTAPHORIQUES POUR UN PROCESSUS ANTHROPOPOIÉTIQUE}

Mais assez des résumés mythographiques. En suivant la documentation classique très fragmentaire que nous avons sur la figure d’Érichthonios, il convient de retracer les valeurs symboliques attachées aussi bien aux protagonistes des récits de fondation quaux acteurs des pratiques rituelles, divinités et fidèles; et ceci, pour illustrer la perspective annoncée, en rapport avec les espaces cultuels, avec leur dimension paysagère, dans lesquels ils évoluent pour être soumis à un processus d’anthopopoiésis identitaire.

\section{I. RÉCIT HÉROÏQUE Enfant et jeunes filles}

L’histoire, par bribes, nous est rappelée par la tragédie aussi poétique que politique qu'est l'Ion d'Euripide. Le jeune Ion n'est autre que le fils naturel né des amours de la plus jeune des filles d’Érechthée, Créuse, et du dieu de Delphes, Apollon. Dans le prologue de la tragédie, le dieu de Delphes nous rappelle comment l'enfant a été exposé par sa mère, jeune fille ; à propos de cette exposition en un lieu qui correspond à la falaise nord-orientale de l'Acropole et qui est dénommé «les Grandes Roches», le dieu évoque en ces termes la naissance d’Érichthonios «né de la terre» (gegenés) :

Car la fille de Zeus avait placé auprès du nouveau-né deux serpents en tant que gardes du corps invitant les Aglaurides, des jeunes filles, à en prendre soin.

De là [hóthen] vient la coutume pour les descendants d’Érechthée, en cette terre, d'élever les enfants au milieu de serpents d'or finement ciselés ${ }^{12}$.

12 Euripide, Ion 21-26; quant à la topographie cultuelle de la falaise nord-orientale de l'Acropole dénommée «Grandes Roches», voir encore infra note 34. 
Dans un premier échange entre Ion et sa mère Créuse (qui identifie sa «terrepatrie » avec la cité d’Athènes), on apprend encore qu'Érichthonios a été accueilli au moment de sa naissance de la terre par Athéna elle-même, la fille de Zeus ; la déesse recueille le bébé dans ses bras de vierge, «elle qui ne l'avait pas engendré ». Puis la déesse confia le nouveau-né, dérobé à la vue, aux filles de Cécrops et d'Aglauros. Les jeunes filles ne purent s'empêcher d'ouvrir la corbeille de la déesse et elles payèrent leur désobéissance d'un accès de folie au cours duquel elles se précipitèrent du haut de l'Acropole. Et Ion d'enchaîner sur le sacrifice par Érechthée de ses propres filles, les sœurs de Créuse, pour sauver la patrie à l'occasion de la guerre contre le roi Thrace Eumolpe; et de citer la mort «autochtone» du roi d'Athènes sous les coups du trident de Poséidon, dans ce même lieu énigmatique des Grandes Roches ${ }^{13}$. Il appartiendra enfin au groupe choral des servantes (athéniennes) de Créuse d’évoquer encore une fois ces Grandes Roches qu'elles situent dans la proximité de la grotte de Pan. En leur sommet, non loin de la grotte de Pan où le petit Ion fut exposé, face aux temples consacrés à Athéna Pallas, sont chantées les filles de Cécrops et d’Aglauros; de leurs pas de danse chorale elles foulent une étendue de gazon au son de la syrinx ${ }^{14}$.

Or c'est non plus à Euripide, mais à Pausanias lui-même que nous devons, sous forme mythographique, le détail du récit de la courotrophie d’Érichtonios : «on affirme qu'Athéna confia Érichthonios à Aglauros et à ses sœurs Hersé et Pandrosos ; la déesse l'avait placé dans un coffre en leur interdisant de s’intéresser à son contenu; on raconte que Pandrosos obéit, mais que les deux autres ouvrirent le coffre, qu'à la vue d'Érichthonios elle furent saisies de folie et qu'elles se jetèrent du haut de la falaise la plus raide de l'Acropole ». Dans son résumé du récit Pausanias, dans une perspective étiologique, rend compte non pas de l'existence

13 Euripide, Ion 258-285. L'histoire tragique de la mort d'Érechthée a été mise en scène par Euripide dans son Érechthée: voir en particulier le fr. 370 Kannicht. Sur les différentes versions de la guerre légendaire conduite contre Érechthée par Eumolpe, le roi de Thrace ou le souverain d'Éleusis, voir Parker, 1987, p. 200-204; voir aussi infra note 35.

14 Euripide, Ion 260-284 et 492-509; les «temples de Pallas» correspondent sans doute, en cette fin du ve siècle, au Parthénon et à l'Érechthéion. La situation topographique de la Grotte de Pan est précisée par Hurwit, 1999, p. 38 (avec fig. 7); pour les prairies de la séduction entre récits héroïques de séduction et pratiques rituelles, voir Calame, 2009 (1996), p. 209-238. 
du sanctuaire héroïque consacré à Pandrosos, mais de la fondation du témenos réservé à sa sœur Aglauros. Quant au sanctuaire réservé à Pandrosos, le Périégète le visitera plus loin pour nous raconter l'étrange rituel de l'arrhéphorie'5.

\subsection{PRATIQUES RITUELLES Les protagonistes de l'arrhéphorie}

Quoi qu'il en soit, assorti de son récit étiologique, le rituel, on se l'imagine, a déjà fait l'objet de commentaires nombreux, anciens et modernes ${ }^{16}$. Pour faire bref, rappelons que, dans la perspective de l'anthropologie contemporaine de la religion grecque, on s'est volontiers appuyé sur l'insertion du service de l’arréphorie dans la séquence des quatre moments rituels marquant la carrière adolescente de l'Athénienne idéale pour donner de ce parcours une interprétation initiatique. Ce parcours anthropopoiétique en quatre étapes est évoqué par les choreutes de la Lysistrata d'Aristophane; de ce parcours rituel les femmes du chœur semblent donner une interprétation initiatique : dès l'âge de sept ans arrhéphore, à dix broyeuse de grain pour l'Archégète, ensuite ourse portant la robe de safran aux Brauronies, enfin canéphore avec le statut de la «belle jeune fille» - qui signifie pour la jeune femme l’accession à la maturité érotique de la puberté achevée ${ }^{17}$. Ainsi, du côté de la pratique rituelle, les objets secrets transportés par les arrhéphores, le passage par un couloir sombre et sauvage, la transition d'un lieu de culte consacré à une déesse vierge vers un sanctuaire consacré à la divinité de la sexualité adulte semblent indiquer un itinéraire d'ordre initiatique, avec ses trois phases canoniques de séparation, de marge et d’agrégation. Du côté de la légende héroïque, la découverte prématurée d’un

15 Pausanias 1, 18, 2; voir aussi Pseudo-Apollodore, Bibliothèque 3, 14, 6; autres versions de la légende chez Burkert, 1966, p. 10-12. Sur le sanctuaire consacré à Aglauros, voir infra note 27. Le riche dossier iconographique, centré sur la naissance chthonienne d'Érichthonios et qui montre en général Athéna casquée, est déployé et commenté par Brulé, 1987, p.68-79; voir aussi Kron, 1981, p.287-290.

16 Les différentes interprétations du rituel décrit par Pausanias 1, 27, 3 sont résumées par Brulé, 1987, p. 83-90; voir aussi Calame, 2009 (1996), p. 232-237.

17 Aristophane, Lysistrata 636-647; sur ce passage au texte quelque peu incertain et à l'interprétation controversée, voir en dernier lieu les remarques décisives de Perusino, 2002; sur les différents statuts évoqués dans ce texte, voir les excellentes remarques de Parker, 2005, p. 218-228. 
nouveau-né, l'accès de folie infligé par la divinité à des jeunes filles et leur mort par précipitation évoquent aussi les morts adolescentes que mettent en scène les récits mis en relation avec les rites de passage réservés aux adolescents et adolescentes. Tout dans le rituel comme dans le récit semble donc inviter l'anthropologue historien de la religion grecque à placer l'arrhéphorie athénienne sous l'étiquette du rite de passage et, plus précisément, sous celle du rite d’initiation tribale, avec sa fonction éminemment anthropopoiétique ${ }^{18}$.

Néanmoins plusieurs informations, entre récit étiologique et gestes rituels, devraient prévenir l'herméneute anthropologue d'insérer le service de l'arrhéphorie parmi les rites qui marquent le passage collectif des adolescents ou des adolescents intégrées à une classe d'âge au statut d’adulte (en tant que membres de plein droit de la communauté). À ce titre on relèvera les éléments suivants : le très jeune âge rituel des arrhéphores qui se situe entre 7 et I I ans, le nombre restreint des filles prépubères et de bonne famille qui sont choisies par l'archonteroi pour ce service religieux, le retour des jeunes filles sur l'Acropole avant de quitter définitivement le service d’Athéna; s’y ajoute, dans le récit héroïque, la mort des Cécropides qui fixe le statut des deux jeunes filles désobéissantes au moment d'une adolescence encore immature. En revanche, d'ordre anthropopoiétique, la courotrophie narrative du nouveau-né Érichthonios, qui est destiné à devenir en tant qu'Érechthée l'un des rois légendaires et fondateurs les plus marquants de l'Attique, et la participation rituelle des arrhéphores à la préparation de la fête nationale en faveur de la déesse maîtresse de la cité engagent à une interprétation d'ordre politique. Une telle lecture est encouragée par le rôle de réciprocité métaphorique assumé d’une part par la sexualité humaine et d’autre part par le processus de croissance de la végétation; il se manifeste dans les deux faces, narrative et rituelle, de la manifestation symbolique comme processus anthropopoiétique certes, mais pas forcément d’ordre initiatique. 
C'est l'interprétation indigène qui nous invite dans une telle direction. Avec sa triple orthographe (arrhé-/errhé-/(h)ersephoria) la dénomination du rituel a été mise en relation étymologisante, déjà par les Anciens, d'une part avec le caractère indicible et mystérieux des objets transportés, d’autre part avec le nom de la fille de Cécrops, Hersé, qui correspond au nom de la rosée ${ }^{19}$. Par ailleurs, un commentateur tardif indique que le rite auquel il donne le nom d'Arrhétophories a pour but de favoriser aussi bien la production des fruits que la procréation des êtres humains : il précise qu’à cette occasion, «on rapporte à la surface des objets sacrés et indicibles (árrẹta) faits de suif et de farine de blé, imitant des serpents et des organes masculins $\gg^{20}$. Serait-ce à dire que, plutôt qu'à un rituel d'initiation tribale, l'Arrhéphorie correspond à l'un de ces «festivals de fertilité » chers aux historiens des religions du XIX ${ }^{e}$ siècle? Sans doute, par figures héroïques et métaphores interposées, l’analogie entre la productivité de la terre et l'engendrement des humains est-elle inscrite dans la séquence athénienne des «mythes d'autochtonie», mais de manière réciproque: la terre productrice parce qu'elle a été fertilisée et cultivée est comparée à la femme qui engendre et qui élève, dans un va et vient métaphorique déjà évoqué et que l'on aura encore à préciser $^{21}$ !

19 Ces différentes explications étymologisantes figurent dans différentes notices dont la référence est indiquée par Brulé, 1987, p.79-83 (également sur l'âge des filles), avec en particulier le témoignage de la scholie à Aristophane, Lysistrata 64I (II. 4, p.64I Holwerda) qui remonte à l'Atthidographe Istros, FGrHist. 334 F 27; voir aussi, à propos des données lexicographiques, Donnay, 1997, p. 178-186. Sur le nom de Hersé à laquelle les inscriptions substituent la figure de Courotrophos (voir infra note 33), voir Brulé, 1987, p. 30-43.

20 Scholie à Lucien, Dialogue des courtisanes (p. 276, 13-17 Rabe); par trop centrée sur le rite de fertilité, la comparaison entre les gestes rituels des Arrhéphories, des Scirophories et des Thesmophories athéniennes est conduite par Deubner, 1932, p. 13-15 et p.40-44, sur fond de rite de fertilité (voir les remarques critiques de Burkert, 1966, p. 5-9); on se référera aussi à Parker, 2005, p. 173-177 et p. 221-224.

21 Voir Platon, Ménéxène 237d-238a: «car ce n'est pas terre qui a imité la femme dans la grossesse et l'enfantement, mais la femme la terre»; le texte de Platon est interprété par Loraux (1996, p. 132-135) dans une lecture littérale que corrige opportunément Georgoudi (2002, p. 131-133). 
Mais dans le contexte de la représentation mythique et rituelle de l'autochtonie athénienne en relation avec les filles de Cécrops, il convient d’abord de tenir compte de la nature double d'un père premier roi de l'Attique : l'ophidien Cécrops «qui déroule ses replis auprès de ses filles», comme nous le rappelle Euripide dans l'Ion, toujours en cette même fin du v ve siècle athénien. Dans cette conjoncture culturelle le serpent est appréhendé en tant que «fils de la terre» (civique) comme le confirme l'histoire de la prise de Sardes telle que la rapporte Hérodote; la destruction de la ville de Crésus est annoncée par une apparition de serpents rapidement mangés par les chevaux qui paissaient dans les faubourgs; consultés, des devins de Carie voient dans les serpents, fils de la terre, les habitants de Sardes et dans les chevaux, animaux belliqueux venus du dehors, les soldats de Cyrus ${ }^{22}$. Par naissance de la terre interposée le serpent peut donc devenir l'homologue du citoyen autochtone. Cécrops, le premier roi autochtone de l'Attique, arbore une queue de serpent; le petit Érichthonios né de l'union différée d’Athéna et d'Héphaïstos par le biais de cette même terre a pour premiers gardes du corps deux serpents; son petit-fils Ion, déposé dans une corbeille analogue, porte sans doute les serpents d’or qui désormais identifient les Athéniens, «fils d’Érechthée».

Aux affinités du serpent avec la naissance des entrailles de la terre il convient d’ajouter la valeur fertilisante que les Grecs attribuaient, à travers différents récits, à l'eau tombant du ciel pour pénétrer le sol: humidité fécondante, sous différentes formes parmi lesquelles la pluie de Zeus ou le sperme d’Héphaïstos; première anthropopoiésis d'ordre végétal. La réciprocité métaphorique entre le végétal et l'humain est à nouveau entière : pluie d'or de Zeus qui métaphoriquement féconde Danaé pour donner naissance à Persée, semence d'Héphaïstos qui fertilise littéralement la terre attique d'où sortira Érichthonios; en poète savant, Callimaque qualifie l'enfant de «rosée d’Héphaïstos ${ }^{23}$ ! Cette fertilité du sol par l'élément humide qui le nourrit, deux des Cécropides en tout cas la portent indirectement

22 Voir Euripide, Ion II63-II64, puis Hérodote I, 78, 3; à propos des valeurs «chthoniennes》 attribuées au serpent en Grèce classique, voir Gourmelen, 2004, p.38-48, p. 329-349 et p. 393-400.

23 Selon le récit repris par exemple par Simonide fr. 543 Page et par Pindare, Pythique 12, 15-17; voir Callimaque, fr. 260, 19 Pfeiffer. 
dans leur nom propre: Hersé, Goutte d'eau, pour cette rosée qui est boisson et aliment pour les cigales faisant résonner l'été de leurs chants ou qui dans sa fraîcheur fait croître les arbustes de même que la poésie d'éloge grandit la valeur²4; et Pandrosos, Toute Rosée, pour cette même rosée à laquelle on compare la grâce éclatante des Charites ou le tendre duvet qui, avec la croissance adolescente, couvre la joue des jeunes gens ou leurs parties... ${ }^{25}$ Si eérse nous renvoie au vaste champ métaphorique de la croissance et de la floraison végétales grâce à l'écoulement d'une eau fraîche et à l'irrigation, drósos nous invite plutôt dans celui de la délicatesse lumineuse des végétaux couverts de rosée. D’une naissance anthropopoiétique masculine (de l'ordre du récit) on est passé à des adolescences féminines, l'une et les autres chargées des valeurs métaphoriques de la croissance végétale.

Quant à Aglauros, Brillance, elle porte souvent, par métathèse, le nom de sa mère Agraulos, la Champêtre, l'épouse de Cécrops; comme la Charite Aglaia, la resplendissante Cécropide affiche dans son nom la beauté éclatante à laquelle renvoie la qualificatif aglaós, en combinaison étymologique possible avec un mot qui renverrait à l'eau courante et qui l'associerait ainsi à chacun des noms de ses deux sœurs ${ }^{26}$. D’Aglauros on sait qu'elle était la première nommée au terme du serment que les éphèbes prêtaient à l'âge de $\mathrm{i} 8$ ans dans le sanctuaire même qui lui était consacré au pied nord-oriental de l'Acropole.. ${ }^{27}$. Dans le texte du serment datant du Iv ${ }^{\mathrm{e}}$ siècle, ces jeunes recrues, futurs citoyens d'Athènes, sont étroitement associés aux puissances de la fertilité et de la productivité agricole

24 Pseudo-Hésiode, Bouclier 393-396; Pindare, Némeénne 8, 40-41; autres références et explications étymologiques chez Boedeker, 1984, p. 52-79.

25 Pindare, Isthmique 6, $62-64$ et Aristophane, Nuées 977-978; cf. aussi Anacreontica 17, 9-11; autres exemples, notamment relatifs à l'inspiration poétique, chez Nünlist, 1998, p. 186-196.

26 On verra à propos de l'incertitude morphologique régnant sur le nom d'Aglauros/Agraulos et sur les spéculations étymologisantes qu'elle a suscitées l'analyse détaillée proposée par Brulé, 1987, p.28-31. On notera que les manuscrits de l'lon d'Euripide orthographient en général le nom de l'épouse de Cécrops comme Agraulos (voir vers 23 et 496).

27 Inscription d'Acharnes: 204 Tod; Aglauros/Agraulos est prise à témoin non seulement avec Hestia, Athéna, Zeus et différentes divinités de la guerre, mais aussi avec Thallô et Auxô, incarnations divines de la floraison et de la croissance, Hégémoné, une Charite, et avec les blés, les orges, les vignes, les oliviers et les figuiers; voir aussi la liste de divinités donnée par Pollux 8, 105; voir Pélékidis, 1962, p. II0-113, ainsi que Siewert, 1977, p. 109-III, qui relève les traits linguistiques et les éléments de culte qui pourraient faire remonter ce texte à l'époque pré-classique. 
du sol et du territoire politique dont ils sont appelés à défendre les frontières. Processus d’anthropopoiésis identitaire non plus narrative, mais rituelle pour les filles, mais également pour les jeunes gens.

\section{ESPACES CULTUELS ET PAYSAGERS}

Du point de vue cultuel, Pandrosos disposait donc face à l'Érechthéion du petit sanctuaire héroïque qu’on a déjà mentionné et qui était inclus dans l'enclos où croissait l'olivier sacré. Rappelons que cet olivier miraculeux évoque, dans la lutte pour la possession divine de l'Attique, le geste de plantation arboricole d’Athéna sur le roc infertile de l’Acropole; il constitue la réponse donnée par la déesse poliade au coup du trident de Poséidon et au jaillissement non moins miraculeux de la source d'eau marine dont les traces étaient vénérées dans l'Érechthéion lui-même. Pandrosos, Toute Rosée, la jeune fille obéissante dont on peut supposer qu’elle a assumé la courotrophie d'Érichthonios, est donc la voisine cultuelle directe d’Athéna Polias ${ }^{28}$. Dans ce voisinage courotrophe la déesse est donc représentée par l'olivier éternellement vert et brillant, l'«olivier de la cité». Cet arbre sacré renvoie, dans son constant renouvellement, à la part prise par la déesse non seulement à la productivité alimentaire de l'Attique, mais aussi à l'éducation guerrière des jeunes pousses que sont les éphèbes ${ }^{29}$ : métaphore de l’anthropopoiésis masculine dans la construction rituelle et symbolique de l'identité du citoyen-soldat. D’autre part, par l'intermédiaire du récit étiologique, serpent et rosée associent les très jeunes arrhéphores et leurs gestes rituels à l'engendrement et à la naissance (autochtone !) des futurs citoyens athéniens ${ }^{30}$. Un tel engendrement n'est possible, dans l'union sexuée, que par l'intervention d'Éros, incarnation d’un désir amoureux qui se manifeste cultuellement dans les jardins réservés à Aphrodite ${ }^{31}$.

28 Voir Hurwit, 1999, p. 204-205, et Parker, 2005, p.51-52 et p. 219-222. Sur les témoignages qui, par le jeu des épiclèses, associent non seulement Pandrosos mais aussi Aglauros à Athéna, voir Kearns, 1989, p.23-27 (avec note 8I).

29 Lire en particulier Sophocle, Edipe à Colone 701-706, pour une «configuration politico-religieuse» reconstruire avec abondance et acuité par Detienne, 1989, p.7I-84.

30 Voir Calame, 2009 (1996), p.232-237. Plutôt que de «initiation into womanhood», Kearns (1989, p. 24-26) pense à un rite centré sur «childbirth and childcare».

31 Quant au rôle joué par Aphrodite et Éros dans l'accomplissement du service arrhéphorique, 
L'ensemble topographique et métaphorique que forment les différents sanctuaires, les gestes rituels et les récits associés aux filles de Cécrops, le premier souverain encore mi-animal de l'Attique, est d'une remarquable cohérence : les éléments végétaux sont combinés avec les constructions architecturales pour inscrire dans ce paysage cultuel les valeurs métaphoriques de la génération et de la croissance des citoyens attachées à la fertilité du sol de l’Attique (transposée par le «mythe» sur le roc de l’Acropole!). En relation avec les récits héroïques le complexe rituel s'appuie sur une géographie cultuelle qui reprend les valeurs symboliques inscrites dans les récits étiologiques. À commencer par le sanctuaire consacré à l'obéissante Cécropide Pandrosos; il est adossé à l'Érechthéion luimême paré de l'olivier sacré naguère planté dans le roc de l’Acropole par Athéna. Ainsi spatialement inclus dans le périmètre de l’Érechthéion, le Pandroséion était situé au-dessus du sanctuaire réservé sur le flanc septentrional de l’Acropole à Aphrodite aux Jardins et à son parèdre horticole Éros. Évoquant les arrhéta, indicibles objets rituels portés à la dérobée par les deux petites arrhéphores, les nombreux phallus de pierre retrouvés dans la zone semblent confirmer la relation directe de ce sanctuaire avec la sexualité humaine la plus explicite ${ }^{32}$.

Par ailleurs, l'enclos incluant avec l'olivier d'Athéna le petit sanctuaire consacré devant l'Érechthéion à la vierge Pandrosos se trouve à moins de trente mètres de l'édifice identifié comme la maison des arrhéphores; spatialement, cette modeste demeure peut être mise en relation avec plusieurs lieux de culte qui, adossés à la falaise septentrionale et orientale de l'abrupt rocher de l'Acropole, jouent un rôle dans la légende héroïque, réélaborée à la fin du v siècle. Tout d’abord il y a la grotte de Pan devant laquelle Euripide situe la danse des Cécropides et que l'on a identifiée avec une cavité qui, au pied du rocher, se trouve entre l'aplomb de la maison des arrhéphores et la base septentrionale des Propylées; la grotte est proche de l'endroit où l'on s'imagine que Hersé et Aglauros se sont jetées dans

voir successivement Bérard, 1974, p. 117-125; Pirenne-Delforge, 1994, p.48-59; Calame, 2009 (1996), p. 236-238. De manière plutôt floue, Donnay (1997, p. 200-202) penche pour une «initiation à la sexualité».

32 À partir du premier rapport par Broneer (1932) sur des fouilles qui ont permis d'identifier le sanctuaire, on verra la synthèse circonstanciée offerte par Pirenne-Delforge (1994 p. 48-59); voir encore Hurwit, 1999, p. 41-43. 
le vide. S’il est bien correct de l'identifier avec la figure de Courotrophos, Hersé recevrait sans doute des honneurs héroïques dans la grotte située dans la falaise septentrionale de l'Acropole, immédiatement sous la maison des arrhéphores ${ }^{33}$.

Quant à Aglauros-Brillance, elle faisait l'objet des honneurs dus à une héroïne dans le sanctuaire où était prêté le serment des éphèbes; on situe désormais ce lieu de culte dans une autre anfractuosité située au nord-est de falaise de l’Acropole, sur ce même flanc escarpé où l'on vénérait aussi, avec Éros, Aphrodite en son jardin, en relation avec l'emplacement de l'agora archaïque située à l'est du rocher sacré. C'est par ce sanctuaire que nous avons commencé notre itinéraire architecturosymbolique en anthropopoiésis narrative et rituelle ${ }^{34}$. Au centre géographique de ce dispositif cultuel se dresse dès le dernier quart du v siècle l'Érechthéion. Ce sanctuaire abrite le culte à la fois héroïque et divin rendu au roi Érechthée en association avec Poséidon; et de ce temple son épouse héroïque Praxithéa devient la première prêtresse au service d’Athéna Polias, entre olivier et eau de mer surgissant l'un et l'autre du roc de l'Acropole ${ }^{35}$. L'action narrative dont des filles de Cécrops sont les protagonistes et l'action cultuelle accomplie par les petites arrhéphores qui représentent sans doute les filles des citoyens se déploient donc, de manière anthropopoiétique, autour du sanctuaire qui est le symbole aussi bien des valeurs économiques et civiques défendues par Athéna que des valeurs de l'expansion maritime de la cité incarnées par Poséidon, au cœur de l'époque classique ${ }^{36}$.

33 Quant à l'hypothétique identification de Hersé avec Courotrophos, voir le dossier récemment soumis à un nouvel examen par Pirenne-Delforge, 2004, p. 175-185, à la suite de ses propres considérations de 1994, p.60-62, et de celles de Brulé, 1987, p.41-43. Le sanctuaire probablement consacré à Hersé a été tour à tour situé dans la maison des arrhéphores et dans le sanctuaire d'Aphrodite aux Jardins: voir Kron, 198I, p. 286.

34 La controverse quant à la situation du sanctuaire dédié à Aglauros fut provoquée par la découverte, plus à l'est de la grotte probablement réservée à Hersé, d'une inscription concernant sa prêtresse: SEG XXXIII (1983), II5; voir le commentaire publié dans SEG XLVI (1996), I37; voir Dontas, 1983, et à sa suite, Greco, 1996, p. 623-626, ainsi que Hurwit, 1999, p. 204 (avec fig. 8); sur Courotrophos, Blauté et Aphrodite, voir Pirenne-Delforge, 1994, p.60-62 et 2004, p. 183-184.

35 Le nom de culte Poséidon-Érechthée est attesté dans l'inscription IG I2, $580=$ IG I3. 2, 873 (qui date du milieu du ve siècle). Les martúria de l'histoire fondatrice de l'Attique et d'Athènes qui sont vénérés dans le temple d'Athéna Polias dénommé Érechthéion sont mentionnés par Pausanias (voir I, 26, 5 et 27, I-2) à l'occasion de sa visite de l'Acropole; voir déjà Hérodote 8, 55, ainsi que Parker, 1987, p. 20I-204; cf. aussi supra note 13.

36 Sur la collaboration cultuelle et symbolique d'Athéna et de Poséidon en Attique, voir Calame, 1996 (1990), p. 348-355. 


\section{CONCLUSION Une éducation gynécopoiétique}

Essentiels sont donc les échos métaphoriques que se renvoient réciproquement l'espace paysager d'ordre cultuel avec les valeurs symboliques attribuées aux éléments physiques et végétaux qui le composent, les actes rituels qui s’y déroulent avec les récits héroïques qui les fondent, le statut social de leur protagonistes, les fonctions et champs d'action des dieux et des héros à qui sont consacrés ces actes de culte. Ces êtres divins sont mis en scène dans les «mythes» étiologiques qui légitiment les pratiques rituelles aussi bien par référence au moment axial et spatio-temporel d'une «première fois » que dans un «ici» où la forme poétique, dans la performance musicale, donne toute son efficacité au récit. De ce point de vue d'une anthropologie historique des pratiques religieuses des Grecs en contexte, il conviendrait, en ce qui concerne l'éducation anthropopoiétique de la jeune Athénienne, d’ajouter au service de l'arrhéphorie en tout cas le double culte rendu à Artémis Braurônia, à Braurôn et sur l'Acropole même, ainsi que les différents cultes dédiés à Déméter et à sa fille entre Athènes et Éleusis. Paysagers, les sanctuaires correspondants balisent l'espace religieux d'Athènes tout en accueillant et en consacrant, dans et par le rituel, une série de transitions inscrites dans l'itinéraire biologique et social suivi par l'épouse du citoyen: naissance, éducation, puberté, nubilité, union sexuelle, première grossesse, premier accouchement, courotrophie, décès, dans un processus continu de «gynécopoiésis» comme processus de construction sociale et culturelle de la femme.

Marqué par l'éducation chorale qui conduit la jeune fille à l'éclosion de la beauté et au mariage procréateur dans l’accomplissement du désir érotique, ce cursus sollicite, à travers une série d'actes cultuels relevant en partie de la vaste catégorie du rite de passage, des divinités telles qu'Athéna, Artémis, Aphrodite et Déméter avec leurs parèdres souvent héroïques. Dans une série de correspondances assimilant l'effet fertilisant de l'eau claire, la croissance des végétaux et l'adolescence des mortels d'une part, l'effet du travail agricole, la production des aliments de base et la procréation favorisée par la pulsion érotique d’autre part, la femme athénienne est conduite par la constel- 
lation des divinités qui la protègent dans un itinéraire de vie qui va jusqu’à la promesse eschatologique d'une vie se prolongeant dans l'au-delà. Par le biais de l'éducation chorale et par l'intermédiaire des actes rituels se réalise, dans la géographie physique de l’Attique, un espace configuré en un paysage cultuel et symbolique par l'activité anthropopoiétique des femmes et des hommes. Ses valeurs symboliques sont réactualisées et entretenues autant par les pratiques cultuelles quaccueillent ces espaces de configuration paysagère que par des manifestations poétiques telles les représentations musicales des tragédies d’Euripide, entre narration dramatisée et pratique rituelle.

\section{BIBLIOGRAPHIE}

AFFERGAN Francis et al., 2003, Figures de l'humain. Les représentations de l'anthropologie, Paris, Éditions de l'EHESS.

BÉRARD Claude, 1974, Anodoi. Essai sur l'imagerie des passages chthoniens, Neuchâtel, Institut Suisse de Rome.

BOEDEKER Deborah, 1984, Descent from Heaven. Images of Dew in Greek Poetry and Religion, Chico, Scholars Press.

BONNET Corinne et PIRENNE-DELFORGE Vinciane, 1999, «Deux déesses en interaction: Astarté et Aphrodite dans le monde égéen », in Les Syncrétismes religieux dans le monde méditerranéen antique, Corinne Bonnet et André Motte (dir.), Bruxelles-Rome, Academia Belgica, p. 249-273.

BREMMER Jan N., 2008, Greek Religion and Culture, the Bible and the Ancient Near East, Leiden-Boston, Brill.

BRONEER Oscar Th., 1932, «Eros and Aphrodite on the North Slope of the Acropolis in Athens», Hesperia I, p. 31-55.

BRULÉ Pierre, 1987, La Fille d'Athènes. La religion des filles à Athènes à l'époque classique. Mythes, cultes et société, Besançon-Paris, Annales Littéraires de l'Université de Besançon/Les Belles Lettres. 
BURKERT Walter, 1966, «Kekropidensage und Arrhephoria. Vom Initiationsritus zum Panathenäenfest», Hermes, 94, p. I-25. Repris in Id., 20II, Kleine Schriften V, Mythica, Ritualia, Religiosa 2, Göttingen, Vandenhoeck \& Ruprecht, p. 160-185.

BUXTON Richard, 1994, Imaginary Greece. The Contexts of Mythology, Cambridge, Cambridge University Press.

CALAME Claude, 1996 (1990), Thésée et l'imaginaire athénien. Légende et culte en Grèce antique, Lausanne, Payot.

-, 2002, «Interprétation et traduction des cultures. Les catégories de la pensée et du discours anthropologiques», L'Homme, 163, p. 51-78.

-, 2006a, Pratiques poétiques de la mémoire. Représentations de l'espace-temps en Grèce ancienne, Paris, La Découverte.

-, 2006b, «Jardins d'amour et prairies de l'au-delà. Rencontres rituelles avec les dieux et poésie en Grèce antique», Poétique, |45, p. 25-4I.

-, 2009 (1996), L'Éros dans la Grèce antique, Paris, Belin.

-, 2013, «Le chant choral des jeunes filles à Sparte: cadences poétiques, rythmes rituels, arts musicaux et identité sexuée», in Cahiers de Littérature Orale, «D'un rythme à l'autre», Sandra Bornand et Maria Manca (dir.), 73-74, p. 19-40.

COHEN Ada, 2007, «Mythic Landscapes of Greece», in Roger D.Woodard (dir.), The Cambridge Companion to Greek Mythology, Cambridge, Cambridge University Press, p. 305-330.

DETIENNE Marcel, 1989, L'Écriture d'Orphée, Paris, Gallimard.

DEUBNER Ludwig, 1932, Attische Feste, Berlin, H. Keller.

DODD David B. et FARAONE Christopher A. (dir.), 2003, Initiation in Ancient Greek Rituals and Narratives: New Critical Perspectives, London et New York, Routledge.

DONNAY Guy, 1997, «L'Arrhéphorie: initiation ou rite civique? Un cas d'école», Kernos, 10, p. 177-205.

DONTAS George S., 1983, «The True Aglaurion», Hesperia, 52, p.48-63.

GEORGOUDI Stella, 2002, «Gaia/Gé. Entre mythe, culte et idéologie», in Myth and Symbol I. Symbolic Phenomena in Ancient Greek Culture, Synnove des Bouvrie (dir.), Bergen, The Norwegian Institute at Athens, p. I13-134. 
GOURMELEN Laurent, 2004, Kékrops, le Roi-Serpent. Imaginaire athénien, représentations de l'humain et de l'animalité en Grèce ancienne, Paris, Les Belles Lettres.

GRECO Emanuele, 1997, «Definizione dello spazio urbano: architettura e spazio pubblico», in I Greci. Storia Cultura Arte Società - II. 2. Una storia greca, Salvatore Settis (dir.), Torino, Einaudi, p. 619-652.

HURWIT Jeffrey M., 1999, The Athenian Acropolis: History, Mythology, and Archaelogy from the Noeolithic Era to the Present, Cambridge, Cambridge University Press.

KEARNS Emily, 1989, The Heroes of Attica, London, Institute of Classical Studies.

KRON Uta, 1981, «Aglauros, Herse, Pandrosos», Lexicon lconographicum Mythologiae Classicae, I. I., Zürich/München, Artemis, p. 283-298.

LORAUX Nicole, 198I, Les Enfants d’Athéna. Idées athéniennes sur la citoyenneté et la division des sexes, Paris, La Découverte.

-, 1996, Né de la terre. Mythe et politique à Athènes, Paris, Seuil.

MOTTE André, 1973, Prairies et Jardins de la Grèce Antique. De la Religion à la Philosophie, Bruxelles, Palais des Académies.

NÜNLIST René, 1998, Poetologische Bildersprache in der frühgriechischen Dichtung, Stuttgart/ Leipzig, Teubner.

PADILLA Mark W. (dir.), 1999, Rites of Passage in Ancient Greece: Literature, Religion, Society, Lewisburg, Bucknell University Press.

PARKER Robert, 1987, «Myths of Early Athens », in Interpretations of Greek Mythology, Jan Bremmer (dir.), London-Sydney, Croom Helm/Routledge, p. 187-214. -, 2005, Polytheism and Society at Athens, Oxford, Oxford University Press.

PÉLÉKIDIS Chrysis, 1962, Histoire de l'éphébie attique. Des origines à 31 avant Jésus-Christ, Paris, de Boccard.

PERUSINO Franca, 2002, «Le orse di Brauron nella Lysistrata di Aristofane», in Le orse di Brauron. Un rituale di iniziazione femminile nel santuario di Artemide, Bruno Gentili et Franca Perusino (dir.), Pisa, Edizioni ETS, p. 167-174.

PIRENNE-DELFORGE Vinciane, 1994, L'Aphrodite grecque, Kernos, Suppl. 4, Athènes-Liège. 
-, «Qui est la Kourotrophos athénienne?», 2004, in Naissance et petite enfance dans l'Antiquité, Véronique Dasen (dir.), Fribourg-Göttingen, Academic Press/ Vandenhoeck \& Ruprecht, p. 172-185.

REMOTTI Franco, 2003, «De l'incomplétude», in Affergan et al., 2003, p. 19-74.

SHAPIRO Harvey Alan, 1996, «The Daughters of Cecrops», in Pandora. Women in Classical Greece, Ellen D. Reeder (dir.), Princeton, Princeton University Press, p. 39-48.

SIEVERT Peter, 1997, «The Ephebic Oath of Fifth-Century Athens», Journal of Hellenic Studies, 97, p. I02-III. 